\title{
NEW CONCEPTS FOR COMPACT ACCELERATOR/TARGET FOR BORON NEUTRON CAPTURE THERAPY
}

\author{
J.R. Powell, H. Ludewig, M. Todosow, and M. Reich \\ RECPED \\ Department of Advanced Technology, Brookhaven National Laboratory, Upton, N.Y. 11 B5 C 23 1396
}

\begin{abstract}
Two new target concepts, NIFTI and DISCOS, that enable a large reduction in the proton beam current need epithermal neutrons for BNCT (Boron Neutron Capture Therapy) are described. In the NIFTI concept, high energy neutrons produced by $(p, n)$ reactions of $2.5 \mathrm{MeV}$ protons on $\mathrm{Li}$ are down scattered to treatment energies $(\sim 20 \mathrm{keV})$ by relatively thin layers of $\mathrm{PbF}_{2}$ and iron. In the DISCOS concept, treatment energy neutrons are produced directly in a succession of thin $(\sim 1$ micron) liquid $\mathrm{Li}$ films on rotating Be foils. These foils interact with a proton beam that operates just above threshold for the $(p, n)$ reaction, with an applied DC field to re-accelerate the proton beam between the target foils.
\end{abstract}

\section{INTRODUCTION}

Previous designs for accelerator driven BNCT (Boron Neutron Capture Therapy) systems based on neutrons generated by $(p, n)$ reactions of low energy (few $\mathrm{MeV}$ ) proton beams on lithium targets have required cw beam currents in the range of 20 to 50 milliamps $(1,2)$. Achieving such high current levels will require substantial technical development, and will tend to be expensive - a significant factor in determinung whether such systems will be practical for widespread use in hospitals.

The high beam current requirement is a consequence of the low neutron utilization efficiency in the target - neutron moderating/filtering system. In previous accelerator BNCT designs, only about $1 / 1000$ th to $1 / 200$ th of neutrons produced in the target are actually available for use in the patient treatment zone. (These utilization efficiencies, while low, are still far better than those achieved by BNCT medical reactors whose neutron efficiencies are on the order of $10^{-6}$.)

In these previous accelerator BNCT designs, neutrons are generated at high energies (typical maximum energy is $\sim 800$ $\mathrm{keV})$ and degraded to treatment energy ( $\sim 10$ to $20 \mathrm{keV})$ by elastio collisions with a suitable moderator (e.g., $\mathrm{BeO}$ or $\mathrm{Al}_{2} \mathrm{O}_{3}$ ). The moderator zone is relatively thick, which results in a low neutron utilization efficiency.

Two new concepts are proposed that significantly reduce the distance needed to moderate/filter the neutrons to BNCT treatment energies, with a corresponding increase in neutron utilization efficiency.

The first concept, NIFTI Neutron Intensification by Filtered Iransmission through Iron) initially degrades high energy neutrons from the lithium target by inelastic collisions with fluorine atoms in solid fluoride material $\left(\mathrm{PbF}_{2}\right.$ and/or $\left.\mathrm{BeF}_{2}\right)$. Fluorine is attractive, because it has a yery low threshold energy for inelastic scattering ( $100 \mathrm{keV})$ and a high maximum cross section, 3 barns. In contrast to conventional low $Z$ moderators, neutrons that are down scattered to a few tens of $\mathrm{keV}$ by an

"This work was performed under the auspices of the U.S. Department of Energy.

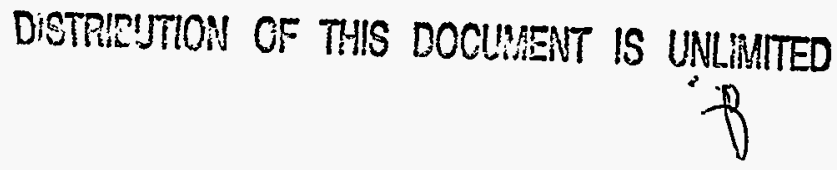

inelastic collision in fluorine do not continue to be further degraded in energy. A relatively thin fluoride zone, on the order of 10 centimeters thick, will efficiently degrade the high energy (maximum of 600 to $800 \mathrm{keV}$ ) neutrons generated in the lithium target.

Located immediately behind the fluoride zone (Fig. 1) is a . layer of iron. Iron has a high (maximum of $\sim 100$ barns) elastic scattering cross section for most of the energy range, from 24 $\mathrm{keV}$ to $\sim 100 \mathrm{keV}$ energies. For neutron energies below 24 $\mathrm{keV}$, however, the scattering cross section has a deep window, with the minimum value decreasing to less than 1 bam. As a result, the NIFTI iron layer strongly impedes the transmission of epithermal neutrons with energies greater than $24 \mathrm{keV}$, until they elastically down scatter into the "window". They then pass through the iron and interact with the patient being treated.

The second concept, DISCOS DISc Configured Orbiting Source) produces neutrons at low energies, typically on the order of a few tens of $\mathrm{keV}$, with a maximum of $\sim 100 \mathrm{keV}$. These neutrons require only a small amount of energy degradation to be useful for BNCT treatment.

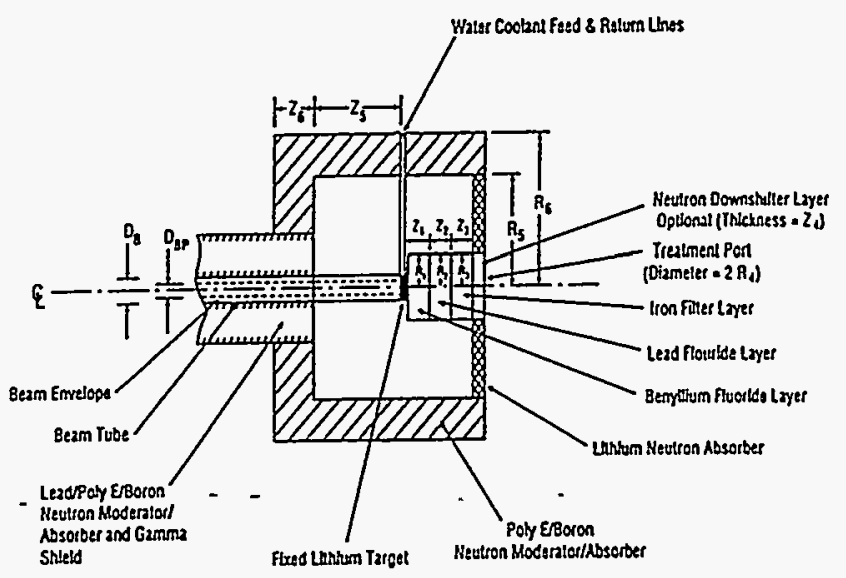

FIGURE 1. Geometry for NIFTI-2/fixed target preliminary designs.

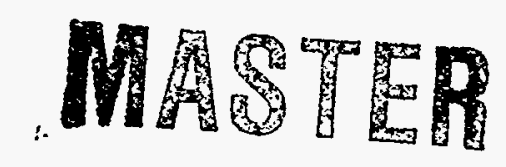




\section{DISCLAIMER}

Portions of this document may be illegible in electronic image products. Images are produced from the best available original document. 


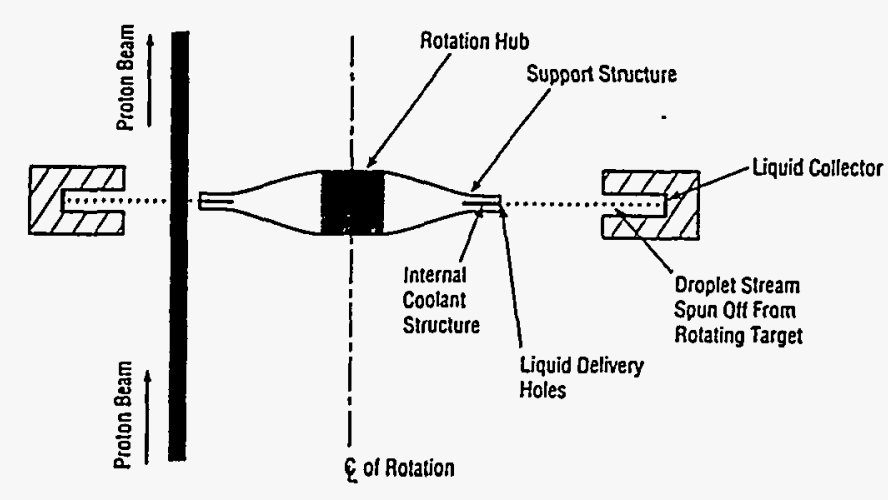

FIGURE 2. Liquid film cooling of DISCOS-1 target.

The DISCOS target consists of a series of very thin rotating beryllium foils that carry thin films ( $\sim 1$ to 2 microns thick) of flowing liquid lithium (Fig. 2). The proton beam impacts and passes through each foil at a nominal energy of $\sim 1.905 \mathrm{MeV}[\sim$ $20 \mathrm{keV}$ above threshold for the $(p, n)$ reaction on lithium]. The energy lost by the protons during their passage through a given foil/film is regained by re-acceleration in a DC electric field that is applied between the foils (Fig. 3). This re-acceleration enables the low energy proton beam to achieve a high overall neutron yield.

The DISCOS target is located immediately behind a relatively thin iron layer, typically about $10 \mathrm{~cm}$ thick, which impedes the transmission of neutrons until they are elastically down scattered into the $24 \mathrm{keV}$ "window". The neutrons can then pass through the iron layer and interact with the patient.

\section{NEUTRONIC UTILIZATION EFFICIENCIES OF THE NIFTI AND DISCOS TARGET CONCEPTS}

An extensive series of neutronic analyses on a range of NIFTI and DISCOS designs has been carried out using the 3D MCNP Monte Carlo neutronics coil. In the NIFTI targets (Fig. 1), the thicknesses and diameter of the $\mathrm{PbF}_{2}$ and iron layers was varied to determine optimum design values. The effect of proton beam energy was also studied, along with the effect of a thin $(\leq 1 \mathrm{~cm}$ ) hydrogenous (water) moderator layer to somewhat "downshift" the neutron energy spectrum at the treatment port.

Table 1 gives the design parameters for representative NIFTI target, and Table 2 gives the corresponding performance parameters for 2 values of proton beam energy, 2.1 and 2.5 $\mathrm{MeV}$. The effect of a neutron "downshifter" ( 1 centimeter of water) located between the fluoride and iron layers is also evaluated [ " $y$ " signifies the presence of the downshifter, " $n$ " signifies no downshifter]. The downshifter functions to downgrade, in a controlled manner, the energy spectrum of the neutrons leaving the fluoride layer.

Approximately 5 to $7 \%$ of the neutron generated in the target exit through the treatment beam port for the cases considered. The beam current required for a desired exit neutron flux of $10^{9}$

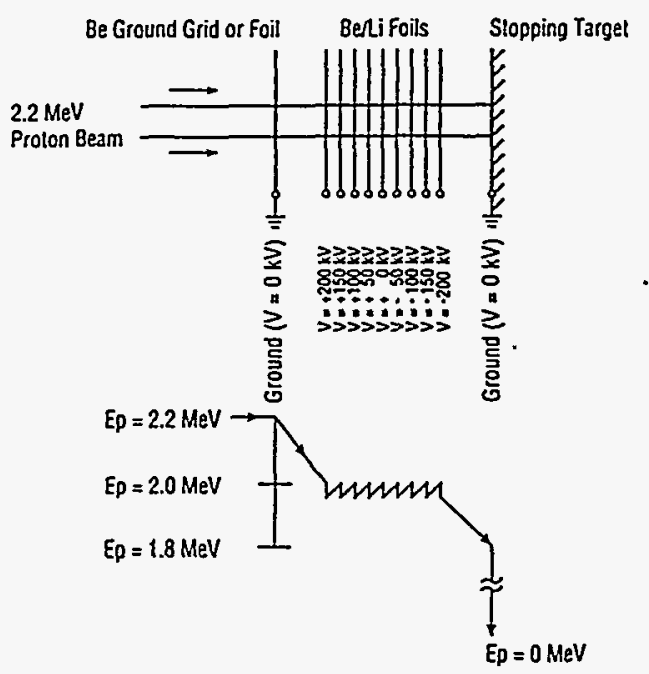

FIGURE 3. Re-acceleration of proton beam by $D C$ fields between multiple foils/films DISCOS-1 target.

$\mathrm{n} / \mathrm{cm}^{2} \mathrm{sec}$ for treatment depends strongly on proton beam energy, ranging from a low value of $\sim 3$ milliamps at $2.5 \mathrm{MeV}$ to $\sim 15$ milliamps at $2.1 \mathrm{MeV}$. This results from the much lower yield of neutrons per incident proton at $2.1 \mathrm{MeV}$. Addition of the neutron downshiffer significantly reduces the average energy of the neutron spectrum, though at some penalty in beam current.

Based on these and other results, it appears possible to design a NIFTI target that can deliver the desired neutron treatment flux at a proton beam current of $\sim 5$ milliamps with an average neutron energy of $\sim 20 \mathrm{keV}$. Such a target would be very attractive for BNCT applications.

Table 1 gives the design parameters for a representative DISCOS target. In this case, the lithium configuration is a series of multiple thin sheets of small diameter droplets (DISCOS-2) rather than being supported as thin liquid films on a series of multiple ultra thin beryllium foils (DISCOS-1). The droplet sheets are located in an applied DC field, with a total voltage of $400 \mathrm{KV}$. The proton beam experiences an average energy loss of $5 \mathrm{keV}$ when it passes through a given droplet sheet; this energy loss is made up by re-acceleration in the DC field between sheets. The beam thus maintains an average energy just above threshold (i.e., $1.902 \mathrm{MeV}$ ) as it traverses the series of 80 sheets. The total useful (i.e., useful for neutron generation) energy imparted to the beam by the $\mathrm{DC}$ field is 400 $\mathrm{keV}$, corresponding to about $20 \%$ of the original input energy of $1.90 \mathrm{MeV}$.

Table 2 gives the corresponding performance parameters for the DISCOS target. Compared to the NIFTI target the fraction of neutrons exiting through the treatment port is considerably greater for the DISCOS target, ranging from $12 \%$ to $19 \%$ depending on whether or not a neutron downshifter is used. This greater neutron efficiency results from the neutron generating zone in the DISCOS target being located closer to the treatment port ( 7.5 centimeters, compared to 15 centimeters 
types. The target materials are readily available, and the target construction is very simple. The beam power load on target is modest, 500 watts $/ \mathrm{cm}^{2}$ for a 5 centimeter diameter beam, and can be readily handled by conventional heat transfer techniques.

Development requirements for DISCOS targets appear to be considerably more challenging. The target must rotate rapidly, on the order of several thousand RPM, either to produce thin flowing liquid films on the foils (DISCOS-1) or liquid droplet sheets (DISCOS-2). In addition, high vacuum conditions must be maintained to prevent electrical breakdown between sheets in the strong applied DC electric field $(\sim 30$ to $40 \mathrm{keV} / \mathrm{cm}$ ).

\section{EFFECTIVENESS OF NIFTI AND DISCOS FOR BNCT TREATMENT}

The effectiveness of a neutron beam generated by an accelerator driven target for BNCT treatment can be assessed using the following criteria:

1. Advantage Depth (AD)

2. Advantage Ratio (AR)

3. Treatment Time

Advantage depth is the depth in tissue at which the total dose to the tumor cells equals the background dose to healthy tissue at the skin surface. It thus measures the effective treatment depth. Advantage ratio is the ratio of total dose to the tumor divided by total dose to healthy tissue, integrated from the surface to the depth of treatment.

Figure 4 compares different BNCT neutron sources with regard to advantage depth and advantage ratio. High values of $A D$ and $A R$ are desirable. The values for $20 \mathrm{keV}, 2 \mathrm{keV}$,

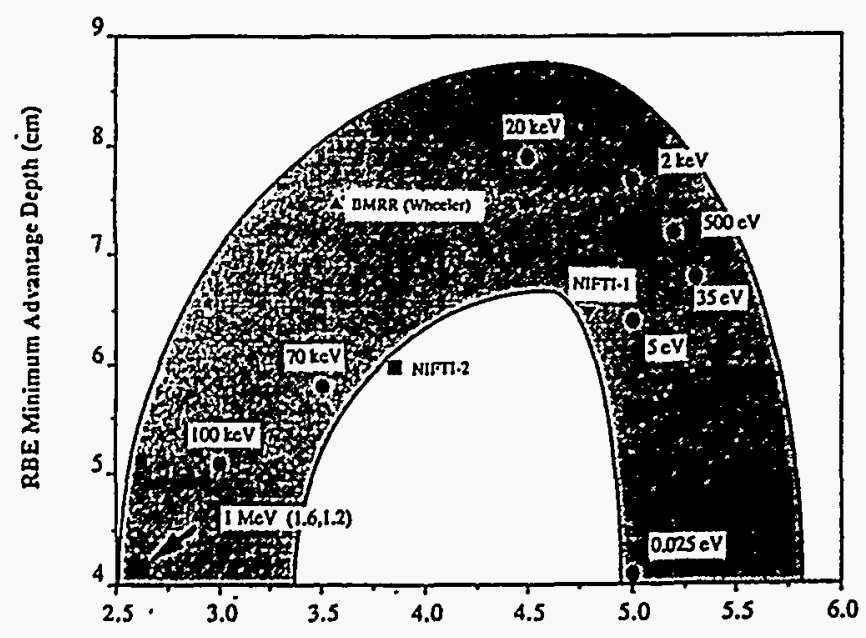

FIGURE 4. Advantage factors for NIFTI-2 and NIFTI-1/DISCOS point designs. etc.(3) refer to ideal monoenergetic, monodirectional neutron beams, while the BMRR value refers to the BNL Medical Reactor. The illustrative NIFTI and DISCOS designs described here appear to have acceptable $A D$ and $A R$ performance. Performance should improve with further optimization, approaching that for an ideal monoenergetic 20 $\mathrm{keV}$ beam. Acceptable treatment times on the order of $1 / 2$ hour appear achievable with a proton current of 5 milliamps.

\section{CONCLUSIONS}

The NIFII and DISCOS target concepts appear to enable practical accelerator driven neutron sources for BNCT treatment at low values of proton beam current, i.e., on the order of 5 milliamps, with acceptable treatment parameters. At such beam current levels, accelerator driven neutron sources would be acceptably low in cost, and could be widely used in hospitals for BNCT treatment. Development requirements for the NIFTI concept appear relatively simple and straightforward; development requirements for DISCOS appear much morechallenging, however. Although DISCOS . offers the possibility of even lower beam currents than NIFTI, its greater development requirements make NIFTI the first choice of the two concepts.

\section{ACKNOWLEDGMENTS}

The authors wish to express their deep appreciation and thanks to Ms. Barbara Roland for editing and preparing the manuscript, and to Ms Judith Otto for preparing the drawings.

\section{REFERENCES}

1. Wang, C.K.C., Eggers, P.E., and Crawford, H.L., "Accelerator Neutron Irradiation Facility for Hospital Based Neutron Capture Therapy," in Advances in Neutron Capture Therapy, Solway, A.H. ed Plenum Press, New York, 1993, pp. 119.

2. Wu, T.H., Brugger, R. And Kunze, J.F., "Low Energy Accelerator-Based Neutron Sources for Neutron Capture Therapy," in Advances in Neutron Capture Therapy, Solway, A.H. ed Plenum Press, New York , 1993, pp. 105.

3. Clement, S.D., et al, "Monte Carlo Methods of Neutron Beam Design for Neutron Capture Therapy at the MIT Research Reactor (MITR-1)," in Neutron Beam Design, Development, and Performance for Neutron Capture Therapy, Hading, O., Bernard, J., and Zamenhof, R., ed Plenum Press, New York, 1989, pp. 51. 